Horizons philosophiques

\title{
Théorie des préjugés selon Descartes et Gadamer
}

\section{Donald Ipperciel}

Volume 7, numéro 2, printemps 1997

L’héritage de l’herméneutique

URI : https://id.erudit.org/iderudit/801043ar

DOI : https://doi.org/10.7202/801043ar

Aller au sommaire du numéro

Éditeur(s)

Collège Édouard-Montpetit

ISSN

1181-9227 (imprimé)

1920-2954 (numérique)

Découvrir la revue

Citer cet article

Ipperciel, D. (1997). Théorie des préjugés selon Descartes et Gadamer. Horizons philosophiques, 7(2), 43-57. https://doi.org/10.7202/801043ar d'utilisation que vous pouvez consulter en ligne.

https://apropos.erudit.org/fr/usagers/politique-dutilisation/ 


\title{
THÉORIE DES PRÉJUGÉS SELON DESCARTES ET GADAMER
}

\author{
"... ayant reconnu que tous ceux qui ont des senti- \\ ments fort contraires aux nôtres, ne sont pas, pour \\ cela, barbares ni sauvages, mais que plusieurs usent, \\ autant ou plus que nous, de raison; et ayant considéré \\ combien un même homme, avec son même esprit, \\ étant nourri dès son enfance entre des Français ou \\ des Allemands, devient différent de ce qu'il serait, \\ s'il avait toujours vécu entre des Chinois ou des \\ Cannibales; [...] en sorte que c'est bien plus la cou- \\ tume et l'exemple qui nous persuadent, qu'aucune \\ connaissance certaine, et que néanmoins la pluralité \\ des voix n'est pas une preuve qui vaille..." \\ Descartes, R., Discours de la Méthode.
}

La philosophie allemande du vingtième siècle est peu élogieuse à l'égard de Descartes. Heidegger, surtout, rejette sur lui l'odieux d'avoir fondé l'ère du subjectivisme philosophique, lequel, telle l'expulsion hors du jardin d'Éden, aurait dépouillé l'homme de son appartenance spontanée au monde vécu, à son Heimat le plus intime. Co-originaire à ce mouvement historial par lequel l'homme devient étranger chez lui, se retrouve sa volonté de devenir «maître et possesseur de la nature. L'homme moderne cherche ainsi à reconquérir par la force ce qui d'abord lui appartenait en propre. Par là s'inaugure l'ère du Ge-stell, de la technique, qui ne trouvera de fin que par un deus ex machina : "Seulement un dieu peut encore nous sauver", affirme-t-il, en accord avec sa pensée du Seingeschick, dans le célèbre entretien du Spieger!

Gadamer, sans faire sien le pathos heideggérien, poursuit l'offensive contre Descartes. II s'en prend surtout à l'illusion scientiste d'un rapport désintéressé à l'objet, pour lequel Descartes est devenu le symbole en tant que père de la moder-

1. Martin Heidegger, Réponses et questions sur l'histoire et la politique, p.49. 
nité. L'absence de préjugés dans une relation épistémique n'est selon Gadamer qu'une construction idéaliste et abstraite qu'une phénoménologie du comprendre peut démasquer. L'herméneutique philosophique, dans la mesure où elle s'est départie de sa tradition instrumentaliste en tant que technique de l'interprétation, et pour autant qu'elle s'est proposée, depuis Heidegger, "de décrire ce qui est»et "de penser ce qui a toujours lieu»?, ne représente rien de moins qu'une telle phénoménologie du comprendre. Sur le fond positif de cette dernière, et négatif d'un rejet de la théorie des préjugés de Descartes et de la modernité, Gadamer cherche à profiler sa propre théorie des préjugés. C'est dans ce cadre que Gadamer propose une réhabilitation des préjugés dans Vérité et Méthode.

À cette réhabilitation des préjugés par Gadamer, nous aimerions répondre par une réhabilitation de la critique cartésienne des préjugés. Cette intention ne s'inscrit pas dans l'esprit général des éloges à l'endroit de Descartes à l'occasion du $400^{\circ}$ anniversaire de sa naissance. Il s'agit ici plutôt de comprendre la théorie des préjugés de Gadamer, ce qui n'est possible qu'en reconnaissant une certaine valeur à celle de Descartes, bien que dans un domaine limité. En faisant justice à Descartes, on fait du même coup justice à Gadamer. On verra que Gadamer ne s'oppose pas véritablement à la critique cartésienne des préjugés: il la sanctionnerait même. En fait, Gadamer ne fait pas la critique de ce que dit Descartes à ce sujet, mais plutôt de ce qu'il ne dit pas.

La thèse centrale de Gadamer dans sa confrontation avec Descartes et la philosophie des Lumières se résume à ceci : il accuse ces derniers d'avoir entretenu un préjugé à l'endroit des préjugés. En réponse à cette faute de la pensée, Gadamer appelle à une réhabilitation des préjugés et de l'autorité. Nous aurons à démontrer dans la suite que les deux termes qui composent l'expression «préjugé contre les préjugés» n'expriment pas une même réalité ou, dans le vocabulaire de la linguistique,

2. Traduction légèrement modifée de: “ich beschreibe, was ist» et "Zu denken, was immer geschieht", ( $\mathrm{HH} 1,2,395,55)$. [Les sigles bibilographiques sont décodés dans la bibliographie] 
le signifiant «préjugé», de par son caractère polysémique, renvoie dans l'expression à deux signifiés différents. L'expression a sans doute été retenue par Gadamer malgré son imprécision parce qu'elle présente une figure de rhétorique intéressante, mais elle n'a l'avantage de préciser ni la position de Gadamer, ni celle de Descartes. Si bien qu'on a proposé dans les traductions anglaises de Gadamer ${ }^{3}$ d'abandonner "prejudice» au profit de "pre-judgement", afin d'éviter tout malentendu. Dans le contexte général de Vérité et Méthode, le choix de la formulation que l'on connaît n'a de sens que dans son effet polémique qui, paradoxalement, se réclame de Descartes lui-même : "L'accroissement de la tension entre vérité et méthode avait, dans ce que j'entreprenais, une signification polémique. Comme Descartes lui-même le reconnaît, il faut, pour redresser un objet tordu, le plier dans le sens opposé" (Gadamer, P. 94; N3A, 453) 4 .

Nous avançons donc, dans un premier temps, la thèse que le préjugé de Descartes, qui s'apparente au sens courant, ne peut s'identifier au préjugé de Gadamer. Pour Descartes, les préjugés sont de deux sortes: d'une part, les préjugés de précipitation (Descartes, $\left.A T_{1}, \mathrm{VI}, 15\right)$ sont des jugements portés avant d'avoir atteint l'évidence; d'autre part, les préjugés de prévention, loin de se restreindre à la seule autorité, comme Gadamer le définit à la suite du père de l'Aufklärung allemande, Christian Thomasius (VM, 109-110; WM, 276), désignent une résistance des hommes à réviser leurs opinions reçues de ce qu'ils sont "gouvernés par [leurs] appétits et [leurs] précepteurs» (Descartes, $A T_{1}, \mathrm{VI}, 13$ ). L'autorité n'est qu'une source possible de la prévention, qui s'identifie plutôt à une propension au dogmatisme ou à l'inertie des idées. Ce n'est pas un hasard si le terme "prévention» prend aussi le sens d' «emprisonnement» en français.

3. Joseph Kockelmans "Towards an Interpretative or Hermeneutic Social Science", Graduate Faculty Philosophy Journal, 5, p.78.

4. "Die Zuschärfung der Spannung von Wahrheit und Methode hatte in meinen Untersuchungen einen polemischen Sinn. Am Ende gehört es, wie selbst Descartes anerkennt, zu der besonderen Struktur des Zurechtbiegens eines verbogenen Dinges, daß man es nach der Gegenrichtung beugen muß". 
De toute évidence, Gadamer ne cherche pas à réhabiliter ce genre de préjugés. Lorsqu'il affirme : «Toute interprétation juste doit se prémunir contre l'arbitraire d'idées spontanées (Einfälle) et contre la limitation des habitudes de pensée (Denkgewohnheiten) non décelées» (Gadamer, WM, 271) ${ }^{5}$, il ne fait rien de moins que s'opposer, comme Descartes, aux préjugés de prévention (Denkgewohnheiten) et de précipitation (Einfälle). Il est donc établi par là qu'on ne peut accuser Gadamer de tomber dans un quelconque romantisme des idées. Force est aussi de reconnaître que Gadamer ne rejette pas en bloc les acquis des Lumières. II ne cherche pas à étouffer l'élan critique que celles-ci ont légué, bien qu'il ne voit pas dans cette pratique l'essence de l'entreprise philosophique.

De l'autre côté, on ne peut attribuer à Descartes l'idée selon laquelle l'autorité et la tradition seraient en soi à proscrire. Non seulement Descartes ne s'est jamais prononcé dans ces termes, mais l'idée même de sa morale "par provision", caractérisée par un respect de l'autorité et de la tradition, s'oppose à un tel jugement. Ceci, Gadamer l'a clairement reconnu (VM, $118 ; \mathrm{WM}, 283)$. Lorsqu'il expose sa résolution de faire table rase de tous ses préjugés, Descartes s'exprime en termes neutres à l'égard de la tradition : "pour toutes les opinions que j'avais reçues jusques alors en ma créance, je ne pouvais mieux faire que d'entreprendre, une bonne fois, de les en ôter, afin d'y en remettre par après, ou d'autres meilleures, ou bien les mêmes, lorsque je les aurais ajustées au niveau de la raison" (Descartes, $A T_{1}, \mathrm{VI}_{1}$ 13-14). Descartes croyait même plus raisonnable de ne pas se lancer inconditionnellement dans cette entreprise sceptique, puisque l'opinion d'une autorité s'avérait dans bien des cas supérieure à celle produite par notre propre jugement : "de ceux qui, ayant assez de raison, ou de modestie, pour juger qu'ils sont moins capables de distinguer le vrai d'avec le faux, que quelques autres par lesquels ils peuvent être instruits, doivent bien plutôt se contenter de

5. Nous traduisons. "Alle rechte Auslegung muß sich gegen die Willkür von Einfällen und die Beschränktheit unmerklicher Denkgewohnheiten abschirmen..."; Cf. VM, 104. 
suivre les opinions de ces autres, qu'en chercher eux-mêmes de meilleures" (Descartes, $\left.A T_{1}, V_{1}, 15\right)$. On le voit bien, les préjugés ne sont pas pour Descartes toujours faux, bien qu'ils soient souillés d'une tache originelle: celle de ne pas avoir été pensés par soi-même, ou pour dire le même, de ne pas être rationnels. Nous reviendrons plus loin sur ce point crucial de la théorie cartésienne des préjugés. Retenons ici que même pour Descartes, un préjugé, suite à l'examen de la raison, peut très bien s' «avérer vrai».

Ainsi, il n'y a pas opposition stricte entre l'esprit des Lumières et Gadamer eu égard à la question des préjugés. Et pourtant, Gadamer cherche précisément à se démarquer de l'Aufklärung en voulant réhabiliter les préjugés. II importe donc de déterminer avec justesse ce que Gadamer entend par "préjugé».

Avec ce dernier, Gadamer reprend un thème heideggérien bien connu, malgré qu'on ne puisse compter ce terme, à titre de terminus technicus du moins, au vocabulaire de Heidegger. II appert cependant que le Vor-urteil gadamérien renvoie à la structure à préalables (Vor-Struktur), laquelle détermine toute ouverture à l'être et donc toute compréhension. Pour Heidegger, l'explicitation objective (sachliche Auslegung) de l'être en tant qu'être, soit la Als-Struktur, présuppose une ouverture préalable à l'être, soit la Vor-Struktur. Cette ouverture préalable révèle l'être à l'homme sous un certain jour, sous une certaine guise. Ce qui peut être articulé dans cette ouverture compréhensive, il l'appelle "sens", et la structuration de ce dernier forme le langage. L'ouverture compréhensive de l'être sera donc de la nature du langage, et possède ainsi virtuellement une forme propositionnelle qui sera réalisée dans l'explicitation. Si je porte un quelconque jugement, celui-ci est toujours, selon Heidegger et Gadamer, précédé d'une pré-compréhension non-explicite qui déterminera mon jugement. Le jugement est donc l'actualisation dans une forme propositionnelle de l'ouverture compréhensive. Parce qu'elles ne sont pas explicites, les thèses pré-propositionnelles de la pré-compréhension ouvrant l'être, elles, ne seront pas connues du sujet 
qui les utilise. Dans le langage du phénoménologue : elles ne sont pas thématiques. La structure compréhensive du sujet est donc portée par des "diathèses", c'est-à-dire des thèses qui traversent tous nos jugements et dans une certaine mesure les jugements d'une culture, sans qu'elles soient perçues d'emblée.

L'apport gadamérien à la théorie heideggérienne de la structure à préalables se trouve dans ceci qu'il ramène à l'unité du préjugé ce qui chez Heidegger avait une forme tripartite (Vorhabe, Vorsicht, Vorgriff). Car le préjugé de Gadamer ne s'identifie pas seulement au Vorgriff 6 , c'est-à-dire à la saisie conceptuelle préalable. II n'est pas seulement un pré-jugé linguistique. De par la structure de renvoi mondain du langage, la Sachlichkeit der Sprache, il va de soi pour Gadamer que l'entièreté de la conjointure (Bewandtnisganzheit), c'est-à-dire le monde, de même que son dévoilement perspectiviste se trouvent d'emblée compris (eingeholt) dans le langage, et donc dans l'idée du pré-jugé.

Le pré-jugé de Gadamer n'est donc pas un jugement préalablement établi par d'autres ou porté avant l'examen définitif qui mène à l'évidence, comme c'est le cas dans l'acception cartésienne et commune du terme. Le pré des préjugés de Gadamer doit être entendu de façon plus radicale que chez Descartes. Le préjugé diathétique de Gadamer, c'est un jugement qui n'en est pas encore un, mais qui peut le devenir en devenant thème de réflexion. Le pré du préjugé indique sa nature ante-prédicative, pré-propositionelle, propre à tout sens non encore explicité. Les préjugés diathétiques se rapportent aux compréhensions implicites qui accompagnent toute saisie du monde, et qui, parce qu'elles sont évidentes et communes, ne sont pas reconnues par la conscience. Ces préjugés, en tant que pré-compréhensions, ont selon Gadamer un aspect productif, parce qu'ils forment la substance même de la compréhension explicite. Celle-ci se constitue toujours, selon l'herméneutique philosophique, sur la base de ceux-là. On est bien

6. C'est le cas notamment de Kögler. Cf. Kögler, H.-H., Die Macht des Dialogs, p. 73 et suivantes. 
loin des préjugés de Descartes qui, eux, de par le dogmatisme et l'arbitraire qui leur sont inhérents, entravent plutôt la réalisation de la connaissance.

Poursuivons cependant l'analyse du préjugé gadamérien afin d'en faire ressortir plus avant le caractère inéluctable. Dans la pensée de Gadamer, la notion de préjugé est intimement liée au concept de tradition. Cette dernière, ce n'est pas le passé de l'antiquaire, c'est-à-dire le passé en tant qu'il est révolu. La tradition s'identifie plutôt à un autre concept-clef de l'herméneutique philosophique, à savoir la situation (VM, 142; WM, 307). Celle-ci est le résultat d'un devenir historial qui se définit par sa structure temporelle, laquelle renvoie avant tout chez Gadamer à un avoir-été. La tradition est donc, selon Gadamer, toujours vivante, en ce qu'elle s'actualise dans la situation herméneutique, laquelle constituera à son tour la structure englobante de nos pré-compréhensions. Situation, tradition et préjugés sont donc du même tissu: ils représentent autant de facettes d'une même réalité, l'éclairant tour à tour de la perspective de l'existence, de l'histoire et du langage. Tous trois expriment la réalité de l'être de sens qu'est l'homme-au-monde ou, dans la terminologie de Heidegger, le Dasein.

Si l'on reconnaît que nous ne pouvons faire abstraction de notre situation - ou pour dire le même : de notre contextualité -, alors on comprend pourquoi il devient absurde pour Gadamer que nous nous défassions complètement de nos préjugés, entendus comme diathèses. Ces préjugés diathétiques sont enracinés dans le sol de la tradition dans laquelle nous nous comprenons toujours déjà. En fait, cette formulation est trompeuse, car préjugés diathétiques et tradition ne font qu'un. Cet «inconscient herméneutique» de la tradition, dynamique en ce qu'il résiste au devenir-conscient ${ }^{7}$, détermine toujours déjà l'homme. Par là reconnaît-on la dimension passive (Geschehen) de notre constitution, sur laquelle Gadamer a

7. “L'expérience herméneutique réalise en effet combien les préjugés peuvent être enracinés profondément et combien une simple anamnèse est impuissante à rompre son emprise". "Die hermeneutische Erfahrung realisiert nämlich, wie tief Vorurteile eingewurzelt sein können und wie wenig ein bloßes Sich-

Bewußtwerden derselben imstande ist, ihre Gewalt aufzulösen" $(\mathrm{KpH}, 115)$. 
tant insisté, et qui trouve sa formulation la plus aiguë dans la citation suivante : «En vérité ce n'est pas l'histoire qui nous appartient, c'est nous qui lui appartenons. Bien avant que nous accédions à la compréhension de nous-mêmes par la méditation réflexive, nous nous comprenons de manière irréfléchie dans la famille, la société et l'État où nous vivons. Le foyer de la subjectivité est un miroir déformant. La prise de conscience de l'individu par lui-même n'est qu'une lumière tremblante dans le cercle fermé du courant de la vie historique. C'est pourquoi les préjugés de l'individu, bien plus que ses jugements, constituent la réalité historique de son être" (Gadamer, VM, 115; WM, $281)^{8}$. L'homme est ici abordé dans sa constitution historiale, pour qui la tradition et les préjugés diathétiques sont déterminants. Ce genre de préjugé reste cependant inconnu pour Descartes, tellement il se fond à l'évidence. Même les «notions communes" ne recoupent pas pleinement, de par l'absence en elles d'une conscience historique, le sens des préjugés diathétiques et situationnels de Gadamer.

II est clair que la formule "préjugé contre les préjugés» emploie un même signifiant pour désigner deux intentions de sens différentes. Le premier renvoie au préjugé du sens commun, celui de Descartes, alors que les deuxièmes désignent les préjugés diathétiques et situationnels, sous-jacents à toute connaissance explicite. Si les acceptions de "préjugé», telles qu'elles sont employées chez Gadamer et Descartes ne se recoupent pas, quel est donc le sens véritable de la critique de Gadamer à l'endroit de Descartes et du projet de réhabilitation des préjugés qui en découle? Pour répondre à cette question, il faut d'abord se pencher sur l'attitude de Descartes face aux préjugés.

8. "In Wahrheit gehört die Geschichte nicht uns, sondern wir gehören ihr. Lange bevor wir uns in der Rückbesinnung selber verstehen, verstehen wir uns auf selbstverständliche Weise in Familie, Gesellschaft und Staat, in denen wir leben. Der Fokus der Subjektivität ist ein Zerrspiegel. Die Selbstbesinnung des Individuums ist nur ein Flackem im geschlossenen Stromkreis des geschichtlichen Lebens. Darum sind Vorurteile des einzelnen weit mehr als seine Urteile die geschichtliche Wirklichkeit seines Seins". 
Le rejet des préjugés par Descartes s'inscrit dans l'entreprise plus générale du doute méthodique. En mettant en doute toutes les opinions qu'il avait jusque-là reçues en sa créance, Descartes s'assurait de parvenir à des vérités "certaines". Le doute méthodique et le rejet des préjugés, en tant que ceci représente une forme d'actualisation du doute, avaient donc pour fin l'accès à la certitude. Cette dernière n'est cependant pas le véritable moment efficient de l'entreprise cartésienne. La morale provisoire de Descartes se fonde non pas sur des certitudes, mais sur la vraisemblance. Ladoption de cette vraisemblance est cependant raisonnable, et c'est la raison qui dicte son adoption résolue. II serait en effet irrationnel de suspendre ses actions, tel l'âne de Buridan, parce que le Vrai n'a pas encore été saisi. "Les actions de la vie ne souffrant souvent aucun délai, c'est une vérité très certaine que, lorsqu'il n'est pas en notre pouvoir de discerner les plus vraies opinions, nous devons suivre les plus probables" (Descartes, $\left.\mathrm{AT}_{1}, \mathrm{VI}, 25\right)^{9}$. La raison, qui ne parle pas uniquement en certitudes, est donc le principe normatif ultime. Comme la méthode de Platon, à savoir la dialectique ${ }^{10}$, mène au vovo, la méthode de Descartes aboutit à la raison.

Descartes définit la raison comme faculté «de bien juger» $\left(\mathrm{AT}_{1}, \mathrm{VI}, 2\right)$ qui constitue, comme Leibniz l'a reconnu ( $\mathrm{La}$ Monadologie, \$29-\$30), un acte réflexif du sujet. La réflexion se révèle comme présence à soi de l'esprit qui permet, comme dans un tribunal, d'entendre les raisons - pris ici dans le sens de justification - et contre-raisons. Les préjugés sont à proscrire selon Descartes non pas en raison de leur caractère préétabli, mais parce qu'ils n'ont pas subi l'examen de ma pro-

9. Cf. Descartes, AT3, IX, II, 26 : “... si l'action ne souffre aucun délai, la raison veut que nous en choisissions une [une opinion vraisemblable]".

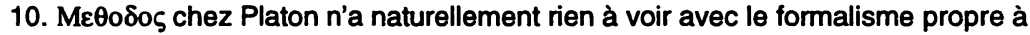
la méthode scientifique cartésienne et post-cartésienne et indique plutôt un procès ou un cheminement vers un certain but. Par ailleurs, plusieurs occurrences dans l'œuvre de Platon confirme le lien entre $\mu \varepsilon \theta 0 \delta$ os et $\delta 1 \alpha \lambda \varepsilon \pi k \eta$; cf. Platon, Resp. VII, 531 d 1, 533 b 3, 533 c 7; X, 596 a 6; Soph. 265 a 2; Leg. XII, 965 c 6 . 
pre raison, parce qu'ils ne sont pas réfléchis ${ }^{11}$. De la même façon, la logique formelle, la syllogistique, est rejetée par Descartes parce qu'elle veut se substituer à la réflexion ${ }^{12}$. II faut comprendre que l'interlocuteur herméneutique de Descartes était l'humaniste, qui, dans l'esprit de Descartes, se contentait d'exercer sa mémoire et d'étaler son érudition sans vraiment saisir la portée de son savoir, et qui ne pouvait que le reproduire ${ }^{13}$. A ce psittacisme, Descartes voulait substituer un savoir vivant qui connaît véritablement ses raisons. C'est en cela que consiste le savoir rationnel, c'est-à-dire le savoir pensé ou réfléchi qui connaît ses fondements.

Gadamer ne peut évidemment que cautionner cette résolution de Descartes à vouloir dépasser l'inanité érudite des humanistes. Par contre, il s'attaquera aux présupposés implicites du rationalisme et du réflexionisme supportent la théorie cartésienne des préjugés. Loin de s'en tenir à un pur mécanisme, le pensée de Descartes possède un aspect téléologique indéniable qu'il importe de dégager dans ce qui suit.

La première cogitation structurante pour la pensée cartésienne est le doute, celle qui permet de trouver le «roc» métaphysique, c'est-à-dire le cogito, sur lequel se construit l'édifice philosophique de Descartes. La deuxième cogitation structurante est l'idée de perfection. C'est elle qui permettra de

11. La raison est la norme ultime qui juge de la légitimité d'une opinion : c'est pourquoi il veut “ ... rejeter tout à fait [chacune] des opinions qui s'étaient pu glisser autrefois en [sa] créance sans y avoir été introduite par la raison” (Descartes, $A T_{1}, V_{1}, 17$ ). Souligné par nous.

12. "... ayant pour principal souci d'éviter que notre raison ne reste en chômage le temps que nous recherchons la vérité sur quelque sujet, nous rejetons ces trop fameuses formes d'argumentation comme contraires à notre propos, et recherchons bien plutôt tous les auxiliaires qui peuvent maintenir notre pensée à l'état d'attention..." (RE, X, 129; $\left.A T_{4}, X, X, 406\right)$.

13. Cf. RE, III, 86; AT $4, X, I I I, 367:$ “... jamais, par exemple, nous ne deviendrons mathématiciens, même en connaissant par cœur toutes les démonstrations des autres, si notre esprit n'est pas en même temps capable de résoudre n'importe quel problème; et nous ne deviendrons jamais philosophes, si nous avons lu tous les raisonnements de Platon et d'Aristote, et que nous sommes incapables de porter un jugement assuré sur les sujets qu'on nous propose; dans ce cas, en effet, ce ne sont point des sciences que nous aurions apprises, semble-t-il, mais de l'histoire". 
prouver Dieu, et par la suite de garantir la réalité extérieure. L'idée de perfection joue cependant un rôle subreptice dans toute la gnoséologie cartésienne. Car si le doute est le moyen de la connaissance, la perfection, elle, en est la fin. Descartes se fixe donc un idéal cognitif, c'est-à-dire la connaissance claire, distincte et transparente à soi, qu'il cherchera à atteindre par la méthode. Cette dernière est dans l'esprit de Descartes en quelque sorte une caution qui lui assure l'accès sans restriction à l'idéal de la pure cognoscibilité : «Mais ce qui me contentait le plus de cette méthode était que, par elle, j'étais assuré d'user en tout de ma raison, sinon parfaitement, au moins le mieux qui fût en mon pouvoir» (Descartes, $\left.\mathrm{AT}_{1}, \mathrm{VI}, 21\right)^{14}$.

La seule limite à la raison et à la réflexion qu'il reconnaît est celle qu'il attribue à l'«humaine condition", c'est-à-dire la finitude de l'homme face à Dieu. La résolution du mystère divin resterait ainsi pour l'homme à tout jamais hors de portée ${ }^{15}$. Cependant, à l'intérieur du domaine accessible par la lumière naturelle, toute limitation de la réflexion est due à des causes extrinsèques qui peuvent être surmontées par une résolution ferme, garantie par une méthode infaillible. Les passions, que représentent les instincts concupiscibles et irascibles, sont certes de véritables barrières pour la réflexion, ce que l'observation de la vie quotidienne confirme inlassablement. Mais pour Descartes, les passions de l'âme ne sont que la contrepartie des actions du corps (Cf. $\mathrm{AT}_{2}, \mathrm{XI}, 328$, Art. 2). Pour dire le même, l'inconscient cartésien, c'est le corps. Les passions de l'âme ne représentent ainsi jamais une limitation intrinsèque de la pensée qui, elle, se trouve en notre complet pouvoir ${ }^{16}$.

L'utopie cognitive de Descartes se comprend à partir de cette conviction. Parce que les cogitations sont la seule chose en notre pouvoir, et que toute limitation est extrinsèque en ce

14. Cf. aussi Descartes, $A T_{1}, V I, 63$ : “Or, ayant dessein d'employer toute ma vie à la recherche d'une science si nécessaire, et ayant rencontré un chemin qui me semble tel qu'on doit infailliblement la trouver, en le suivant, si ce n'est qu'on en soit empêché...".

15. "les vérités révélées [...] sont au-dessus de notre intelligence..." (Descartes, $\left.A T_{1}, \mathrm{VI}, 8\right)$. Cf. $A T_{3}, I X, I I, 36$.

16. "ll n'y a rien qui soit entièrement en notre pouvoir, que nos pensées" (Descartes, $\mathrm{AT}_{1}, \mathrm{VI}, 25$ ). 
qu'elle émerge du corps, il n'en tient qu'à nous, par une résolution ferme pour la méthode, de conduire nos pensées selon la raison. Les pensées ne sont pas toujours libres de facto, mais elles le sont virtuellement. L'autonomie de la raison peut ainsi toujours être réalisée. Cette utopie de la transparence de soi à soi de la réflexion représente le telos de toute entreprise cognitive cherchant à produire des évidences, ou pour dire le même, elle représente la fin de la science.

C'est à cette utopie cognitive que s'en prend Gadamer, sans pour autant vouloir toucher au moment efficient de la critique. II cherche à détruire l'utopie de la pure cognoscibilité de Descartes, dans la mesure où il opère une radicalisation du doute cartésien, lequel porte désormais sur l'évidence même, si claire et distincte soit-elle. En ceci, Gadamer s'inscrit dans le Zeitgeist post-moderne. Non seulement le discours philosophique s'est-il dépouillé de son Dieu17, lequel assurait à Descartes la certitude de toute la réalité à l'exception du cogito (Descartes, $\mathrm{AT}_{3}, \mathrm{IX}, 30$ ), mais le malin génie, qui n'était chez Descartes qu'une hypothèse, hyperbolique de surcroît, s'est ontologisé. Le deceptor cartésien est devenu infrastructure (Unterbau) chez Marx, volonté de puissance chez Nietzsche, processus primaire ou inconscient chez Freud, et trouve une place de choix chez Habermas, comme le montre son expression "communication systématiquement altérée». La philosophie de Gadamer, contrairement à celle de ces herméneutes du caché, est trop irénique pour adopter une quelconque personnification du malin génie cartésien. Mais ce rôle est en quelque sorte joué par la tradition et la langue (en tant que l'autre de la réflexion), dans la mesure où elles obligent l'interprète à douter des évidences qui s'offrent à lui. La tradition détermine toujours une situation vivante qui conditionne l'évidence. L'évidence d'une tradition n'est donc jamais celle d'une autre, l'évidence d'aujourd'hui, jamais celle de demain. On n'échappe pas selon Gadamer à la contextualité du sens.

17. Ou dans les mots de Gadamer : "Dieu s'est dissimulé à nous * et nous vivons dans les ténèbres de Dieu»; "..., daß Gott sich vor uns verborgen hat und wir in der Gottesfinsternis leben” (Gadamer, ÜPZ, 173). 
Pour Gadamer, si la réflexion a ces limites, comme l'indique le titre d'une section importante de Vérité et Méthode (VM, II, II, 3 , a, 185; WM, 346), il faut y remédier par le dialogue herméneutique. Ce dernier permet l'émergence d'autres évidences qui elles aussi porteront la couleur de leur situation, et qui de ce fait ouvriront la possibilité de leur propre dépassement, ad infinitum. Ce "situationnisme» 18 gadamérien démasque toutes les évidences "pérennes» et «immuables» comme autant d'illusions contextuelles. Ainsi, la preuve de l'immortalité de l'âme chez Descartes, pourtant si évidente à ses yeux $\left(A T_{1}, \mathrm{VI}, 59\right)$, ne peut aujourd'hui prétendre convaincre bien des gens.

Le dessein réel de Gadamer dans la question des préjugés devient donc plus clair: expliciter encore une fois la dimension passive (Geschehen) du comprendre, qui s'exprime ici par une limitation du sujet réflexif et de la réflexivité. La théorie gadamérienne des préjugés représente donc une autre facette du leitmotiv de la philosophie de Gadamer, qui cherche à penser la Kehre heideggérienne. Ce motif est explicite chez Gadamer : «Mon herméneutique philosophique cherche précisément à respecter le questionnement du dernier Heidegger et à le rendre accessible d'une nouvelle façon" (Gadamer, ZPD, $10)^{19}$. Ses efforts réitérés pour établir la prééminence de l'être sur la conscience se comprennent ainsi comme la volonté de perpétuer la pensée tardive de Heidegger. Le comprendre n'est pas selon Gadamer principalement action du sujet, mais passion du sujet qui s'est inséré dans un dialogue avec la tradition. Ce serait cependant déformer la pensée de Gadamer que de vouloir lui attribuer la thèse de la complète passivité du sujet et de la supra-subjectivité de la tradition, comme le fait M. Frank (Das individuelle Allgemeine, 1985) et J. Kögler à sa suite (Die Macht des Dialogs, 1992). Car Gadamer charge le sujet-inter-

18. Le choix de ce terme, par opposition à "relativisme", s'impose en raison de l'absence en lui d'une connotation anarchique, comme c'est précisément le cas pour "relativisme". En effet, ce dernier semble sous-entendre un anything goes où l'on est en droit d'affirmer arbitrairement ce que bon nous semble, parce que tout est relatif.

19. "Meine philosophische Hermeneutik versucht geradezu, die Fragerichtung des späten Heidegger einzuhalten und in neuer Weise zugänglich zu machen». 
prète d'une mission à accomplir afin d'accéder au comprendre historique 20 . Mais ceci nous conduit vers un autre débat qu'on ne peut ici développer plus avant.

Du point de vue rhétorique, la formulation «préjugé contre les préjugés", imprécise de par la polysémie de ses termes, fut un succès. On n'a qu'à songer aux nombreux débats portant sur ce thème qui suivirent la parution de Vérité et Méthode. Cependant, elle rend méconnaissable le sens réel de la critique de Gadamer à l'endroit de Descartes. L'analyse qui précède montre que ce dernier fait en quelque sorte office de porte-parole pour toute la modernité. La critique du préjugé cartésien contre les préjugés se dépouille ainsi de son caractère ad hominem. La critique veut plutôt faire ressortir la Seinsvergessenheit, l'oubli de l'être propre à toute la modernité, laquelle surestima la puissance du sujet et négligea de ce fait l'empire opaque de la situation herméneutique qui détermine tout savoir. Gadamer ne fait donc pas la critique d'une thèse particulière chez Descartes, en l'occurrence celle des préjugés. Celle-ci est portée en général par le bon sens et la raison, qui elle aussi reste le principe directeur de la pensée de Gadamer ${ }^{21}$. La théorie cartésienne des préjugés est seulement une occasion pour mettre en valeur ce qui est oublié dans la gnoséologie moderne, à savoir l'aspect supra-subjectif de la tradition. Gadamer ravit au sujet le statut d'unique siège de la vérité, et le réduit à un moment confatal à la tradition dans le procès dialogal qui mène à la vérité. La critique du préjugé contre les préjugés se présente en fin d'analyse comme la critique du préjugé pour le sujet.

Donald Ipperciel

Faculté Saint-Jean, University of Alberta

20. Cf. en particulier, VM, 105 et suiv.; WM, 272 et suiv.

21. Chez Gadamer, la raison n'est pas nécessairement réflexive. Cf. ses concepts de Bewahrung et de phronesis. 


\section{BIBLIOGRAPHIE}

\section{Descartes, René :}

- $\mathrm{AT}_{1}$ : "Discours de la méthode", CEuvres, édition Adam et Tannery.

$-\mathrm{AT}_{2}$ : "Les passions de l'âme", CEuvres, édition Adam et Tannery.

- $\mathrm{AT}_{3}$ : «Les principes de la philosophie», CEuvres, édition Adam et Tannery.

- RE : "Les règles pour la direction de l'esprit", CEuvres philosophiques, édition F. Alquié, Tome I, p. 67-204.

- $\mathrm{AT}_{4}$ : «Regulae ad directionem ingenii», CEuvres, édition Adam et Tannery.

\section{Gadamer, Hans-Georg :}

$-\mathrm{HH}_{1}$ : "Hermeneutik und Historismus", in : Hermeneutik II. Wahrheit und Methode. Ergänzungen. Register., Tübingen : J.C.B. Mohr (Paul Siebeck), 1986, p. 387-424.

$-\mathrm{HH}_{2}$ : "Herméneutique et historicisme», in : L'art de comprendre, Paris : Aubier, 1982, p. 49-87.

$-\mathrm{KpH}$ : «Klassische und philosophische Hermeneutik», in : Hermeneutik II. Wahrheit und Methode. Ergänzungen. Register., Tübingen : J.C.B. Mohr (Paul Siebeck), 1986, p. 92-117.

- N3A : "Nachwort zur 3. Auflage", in : Hermeneutik II. Wahrheit und Methode. Ergänzungen. Register., Tübingen : J.C.B. Mohr (Paul Siebeck), 1986, p. 449-478.

-P: «Postface", in : L'art de comprendre, Paris : Aubier, 1982, p. 89-121.

— UPZ: "Über die Planung der Zukunft", in : Hermeneutik II. Wahrheit und Methode. Ergänzungen. Register., Tübingen: J.C.B. Mohr (Paul Siebeck), 1986, p. 155-173.

- VM : Vérité et Méthode, $2^{2}$ éd., Paris : Seuil, 1976, $346 \mathrm{p}$.

- WM : Wahrheit und Methode, Tübingen : J.C.B. Mohr, 1986, 494 p.

- ZPD : "Zwischen Phänomenologie und Dialektik», in : Hermeneutik II. Wahrheit und Methode. Ergänzungen. Register., Tübingen : J.C.B. Mohr (Paul Siebeck), 1986, pp. 3-23.

\section{Heidegger, Martin :}

- ET : Être et Temps, Paris : Gallimard, 1986, $589 \mathrm{p}$.

- RQ: Réponses et questions sur l'histoire et la politique, Paris :

Mercure de France, 1977, $81 \mathrm{p}$.

- SZ: Sein und Zeit, $16^{\circ}$ éd., Tübingen : Max Niemeyer, 1986, $445 \mathrm{p}$.

Kögler, Hans-Herbert :

Die Macht des Dialogs, Stuttgart : Metzler, 1992, 308 p. 